\title{
Nivel de conocimiento sobre la especialidad de medicina interna y percepción de los usuarios sobre el rol y capacidades del médico internista
}

\author{
Knowledge and perception of users on roles of internal medicine specialty and \\ medical internists
}

\author{
Franco Romaní-Romaní ${ }^{1}$, Fernando Atencia ${ }^{2,3}$ \\ ${ }^{1}$ Médico Cirujano. Facultad de Medicina, Universidad Nacional Mayor de San Marcos, Lima, Perú \\ ${ }^{2}$ Docente de la Facultad de Medicina, Universidad Nacional Mayor de San Marcos. Lima, Perú. \\ ${ }^{3}$ Hospital Nacional Arzobispo Loayza. Lima, Perú.
}

\begin{abstract}
Resumen
Introducción: La especialidad de medicina interna enfrenta muchos retos para permanecer competitiva en el sistema de salud, siendo el escaso conocimiento del público sobre la definición, capacidades y competencias de la medicina interna y del internista uno de esos retos. Objetivos: Determinar cuáles son los conocimientos y la percepción de los pacientes sobre el rol de la medicina interna y del médico internista. Diseño: Estudio transversal, descriptivo y analítico. Lugar: Hospital Nacional Arzobispo Loayza, Hospital Nacional Edgardo Rebagliati y una clínica privada. Participantes: Pacientes adultos que visitaron los servicios de Medicina interna de los tres hospitales. Intervención: Se invitó a llenar un cuestionario anónimo y autoadministrado a los participantes entre el 1 y el 15 de septiembre de 2008. Principales medidas de resultados: Nivel de conocimiento sobre medicina interna e internista, percepción de confianza hacia el internista. Resultados: Quinientos pacientes completaron el cuestionario, 42,8\% del Hospital Loayza, $40 \%$ del Hospital Rebagliati y $17,2 \%$ de una clínica privada. Solo $12,2 \%$ alcanzó un buen nivel de conocimiento. El $79 \%$ de pacientes tuvo percepción de confianza en el internista para el manejo de síntomas, 50,2\% confió en la habilidad clínica del internista y $45,9 \%$ confió en el manejo de las enfermedades específicas. Conclusiones: Los pacientes no tienen una definición clara de la medicina interna y el rol del internista. La percepción de confianza es alta con respecto al manejo de los síntomas, pero disminuye al tratarse de enfermedades específicas y de las habilidades clínicas del internista. Por lo tanto, debe realizarse esfuerzos de educación pública para mejorar el entendimiento del rol del internista como especialista en medicina de adultos.
\end{abstract}

Palabras clave: Medicina interna; internistas; pacientes; confianza.

\begin{abstract}
Introduction: Internal medicine specialty is challenged in remaining competitive in the complex health system. Low public awareness of the capabilities and competence of the internists is one of these challenges.

Objectives: To determine patients' knowledge and perceptions on both internal medicine and medical internist roles.

Design: Transversal, descriptive and analytical study.

Settings: Arzobispo Loayza Hospital, Edgardo Rebagliati Hospital, and a private clinic.

Participants: Adult patients attended at the three hospitals' internal medicine services.

Interventions: The patients solved a self-administered and anonymous questionnaire in September 1-15, 2008.

Main outcomes measures: Level of knowledge on internal medicine and internists and perception of confidence.

Results: Five hundred patients answered the questionnaire, $42,8 \%$ at Loayza Hospital, $40 \%$ at Rebagliati Hospital, and $17,2 \%$ at a private clinic. Only $12,2 \%$ had an adequate level of knowledge; $79 \%$ of patients demonstrated confidence in the internist's ability to treat symptoms, $50,2 \%$ showed confidence in the internist for performing clinical examination and $45,9 \%$ in the internist treating specific diseases.

Conclusions: Patients do not have a clear definition on internal medicine and the role of internists. Therefore, efforts should consider public education on this topic in order to promote better understanding of the internist's role as a specialist in adult medicine.
\end{abstract}

Key words: Internal medicine; internists; patients; trust.

\section{INTRODUCCIÓN}

La especialidad médica de medicina interna enfrenta muchos retos para permanecer competitiva en un cada vez más complejo sistema de salud. Siendo uno de esos retos el escaso conocimiento del público en general sobre las capacidades y competencias del internista ${ }^{(1)}$. A diferencia de la mayoría de especialistas, que claramente son identificados por técnica (por ejemplo, los cirujanos), órgano o sistemas que tratan (cardiólogos) o población objetivo (pediatras), los internistas son confundidos o considerados por la gran mayoría del público como médicos generales e incluso internos de medici- na, desconociendo que se trata de una especialidad, siendo frecuente para los internistas responder a la pregunta ¿exactamente qué significa medicina interna?, ya que la palabra interna o interno sugiere o connota algo misterioso, oculto y hasta desagradable ${ }^{(2,3)}$.

El término medicina interna (inneren medizin), introducido en Alemania por el año 1880, se usó para describir una disciplina de medicina clínica diferente de la cirugía, que se basaba en la fisiología experimental, la química y la bacteriología, más que en el simple seguimiento de la progresión de las manifestaciones clínicas ${ }^{(1,2)}$. De este modo, en Alemania nacieron los términos 'medicina interna' e 'internista'. Una reunión realizada en Wiesbaden, en 1882 , fue denominada por primera vez 'Congreso de Medicina Interna'. Dos años después se crea la revista Boletín de Medicina Interna, que difundía lo que la literatura mundial ofreciera en el campo de la medicina interna ${ }^{(3,4)}$.

En 1982 y 1983, Bean y Friedman, respectivamente, sugirieron que el término internista podría ser confundido con interno de medicina o médico general (conocido en EE UU como general practitioner) ${ }^{(1)}$. Años después, Kurtz, y más recientemente Goldman, en 1997, proponen cambiar el nombre de la especialidad de medicina 
interna a medicina del adulto. ¿Por qué adulto? Para Goldman, la respuesta es simple: los internistas atienden adultos y solo adultos ${ }^{(2)}$.

En una encuesta telefónica realizada por la American College of Physicians-American Society of Internal Medicine (ACP-ASIM), se evaluó la percepción del público sobre la labor de los internistas ${ }^{(1,5)}$. Con los resultados obtenidos, que no fueron nada alentadores, se puso en marcha la 'campaña de relaciones públicas por la medicina interna', cuya frase representativa fue 'Doctores para adultos', la cual puso énfasis en que el internista es el especialista con un estudio de posgrado de tres años en la prevención y tratamiento de enfermedades que afectan a los adultos, entre 35 y 60 años ${ }^{(1,6)}$.

En cuanto a la definición de medicina interna, en el diccionario de la lengua española, no se presenta ninguna, pero sí la hay para internista, "es el médico que se dedica especialmente al estudio y tratamiento de enfermedades que afectan a los órganos internos". Incluso llama la atención que en algunos textos de medicina interna no figure su definición (3). Sin embargo, una definición más integral de medicina interna sería "una especialidad médica que se dedica a la atención integral del adulto enfermo, enfocada al diagnóstico y el tratamiento no quirúrgico de las enfermedades que afectan a sus órganos y sistemas internos, y a su prevención" (3,7-9).

En nuestro medio, no existe información acerca del conocimiento que tiene la población sobre el médico internista y la medicina interna como especialidad; mucho menos, existen estudios acerca de la percepción de los pacientes respecto al rol y capacidad del internista. Aún en poblaciones donde se tiene un mayor desarrollo tecnológico de la medicina y el nivel de conocimiento e información es mayor, existe desinformación y no se tiene una idea clara, completa y segura de lo que es la medicina interna y el internista. En nuestro país, no se ha realizado campañas publicitarias sobre qué es la medicina interna. Por lo tanto, podríamos suponer que el nivel de desinformación es aún mayor, ya que en nuestro medio la gran mayoría del público considera a los internistas como médicos generales y se les niega el carácter de especialista, por desconocimiento ${ }^{(6)}$. El presente estudio busca conocer cuál es el nivel de conocimiento del público en general acerca de la medicina interna y la percepción de los pacientes respecto al rol y capacidades del internista.

\section{MÉTODOS}

Se realizó un estudio transversal, analítico y descriptivo. El tipo de muestreo utilizado fue muestro no probabilístico por conveniencia. Se calculó un tamaño muestral de 398 pacientes, considerando un nivel de confianza de 95\%, 5\% de error absoluto, proporción de conocer lo que es medicina interna de $62 \%$, según un estudio previo ${ }^{(1)}$ y un porcentaje de pérdida de $10 \%$. Participaron pacientes mayores de edad que visitaron los diferentes servicios del Departamento de Medicina Interna de tres hospitales generales de Lima metropolitana: Hospital Nacional Arzobispo Loayza, perteneciente al Ministerio de Salud; Hospital Nacional Edgardo Rebagliati Martins, perteneciente al Seguro Social; y una clínica privada. Para asegurar el tamaño muestral se realizó la recolección de datos entre el 1 y el 15 de septiembre de 2008, invitándose a los pacientes al llenado completo de un cuestionario autoadministrado que buscó determinar cuáles son los conocimientos y la percepción que tienen sobre la medicina interna y el médico internista. Fueron excluidos los pacientes que, a pesar de las indicaciones, se negaron a participar del estudio, los que tuvieron un llenado incompleto del cuestionario y los pacientes analfabetos o con dificultades para la comprensión de las preguntas. Se hizo énfasis en que la participación es anónima y voluntaria, firmando posteriormente el respectivo consentimiento informado cada uno de los participantes.

Luego del periodo de recolección de datos, se captó 500 pacientes de los tres establecimientos de salud, quienes ingresaron al estudio.

Se usó como instrumento de recolección de datos un cuestionario aplicado y previamente validado para el estudio de Salerno y col. ${ }^{(1)}$. Este cuestionario analítico, diseñado para explorar la percepción de los pacientes sobre el rol y las capacidades del médico internista y determinar el nivel de conocimiento sobre la medicina interna y el médico internista, fue sometido a prueba piloto en 20 usuarios del Hospital Loayza, con el objetivo de corregir aspectos del entendimiento de las preguntas, mejorar la presentación del cuestionario y establecer un tiempo propicio para su desarrollo.

El cuestionario consta de tres partes. La primera sección recolecta datos generales como edad, género, nivel educativo y hospital donde normalmente recibe atención. La segunda sección consta de 11 preguntas de opción múltiple (3 opciones): verdadero, falso o no sé; las preguntas estuvieron dirigidas a determinar si los pacientes saben qué es el médico internista, cuál es su campo de acción, cuáles son sus capacidades, duración y carácter de especialidad; diferenciarla de otros términos afines como el de médico de familia, médico general o interno de medicina. La tercera sección estuvo orientada a determinar la percepción de los pacientes sobre las capacidades y ámbito de acción del internista; para ello, se planteó las preguntas según la escala de Likert ( 5 opciones de respuesta) y a su vez fueron divididas en tres partes (síntomas, enfermedades y habilidades clínicas o preventivas), a fin de facilitar el análisis de los resultados y el entendimiento de las preguntas para los participantes. La primera parte sobre síntomas, se pregunta con 6 situaciones; la segunda parte, sobre enfermedades con 13 situaciones; y la parte sobre habilidades para examinar y actividades preventivas, se plantea con 6 situaciones. A los participantes se les pidió marcar con un aspa cada una de las situaciones, de acuerdo a su percepción sobre la competencia del internista.

Para el procesamiento y análisis de la parte de conocimientos, se otorgó un puntaje de ' 1 ' si la respuesta fue respondida correctamente y de ' $O$ ' si la respuesta fue incorrecta o si se marca 'no sabe'. Así, el puntaje máximo fue 11 y el mínimo 0 . Para nuestro estudio, consideramos que un puntaje de 0 a 4 definía un nivel de conocimiento malo, puntaje de 5 a 7 un nivel de conocimiento intermedio y de 
8 a 11 un nivel de conocimiento bueno. Para la parte de percepción sobre las capacidades del internista, cuyas respuestas fueron planteadas según la escala de Likert, se consideró que las respuestas 'muy de acuerdo' y 'de acuerdo' reflejan confianza en el cuidado del internista para esas situaciones, mientras que las respuestas 'ni de acuerdo, ni en desacuerdo', 'en desacuerdo' y 'muy en desacuerdo', reflejan desconfianza en el cuidado del internista.

Se presenta los datos demográficos en forma descriptiva (frecuencia, porcentajes) y se comparó los tres centros hospitalarios usando las pruebas estadísticas de chi cuadrado (género y nivel educativo) y anova (edad).

La parte de conocimientos es presentada en forma descriptiva para el total de pacientes y por centro hospitalario. Se aplicó la prueba chi cuadrado para encontrar asociación entre el nivel de conocimiento y el hospital de procedencia; para el promedio de puntaje entre hospitales se aplicó anova. Para hallar la medida de asociación entre nivel de conocimiento y hospital de procedencia, se recategorizó el nivel de conocimiento como 'bueno' y 'no bueno' (si el nivel de conocimiento fue regular o malo), aplicando odds ratio (OR) entre los hospitales.

Para la parte de percepción, se expresa los resultados como porcentaje de respuestas de los participantes que indicaron percepción de confianza para el manejo de síntomas, enfermedades y habilidades clínicas. Se realizó chi cuadrado para determinar asociación entre las frecuencias de confianza para cada situación con el hospital de procedencia. Luego del análisis bivariado, se obtuvo $O R$ ajustados mediante análisis multivariado por regresión logística para género, nivel educativo, hospital de procedencia y nivel de conocimiento, teniendo como variable dependiente la percepción de confianza para síntomas, enfermedades y habilidades clínicas; para esto, se recategorizó a los pacientes que en las situaciones de síntomas y habilidades clínicas tuvieron de 4 a 6 respuestas que indicaron percepción de confianza, considerándolos como pacientes con confianza hacia el internista para el manejo de síntomas y para su habilidad clínica; de la misma forma los pacientes que tuvieron de 7 a 13 respuestas que indicaban confianza para las enfermedades, se les consideró como paciente con confianza hacia el internista para el manejo de enfermedades. Por último, se realizó algunas asociaciones importantes entre las preguntas de conocimiento y la percepción de confianza hacia el internista, mediante chi cuadrado y OR. Se consideró datos estadísticamente significativos para los valores de $p<0,05$. Se utilizó para el análisis estadístico el programa SPSS para Windows versión 16.

\section{RESULTADOS}

Durante el periodo de recolección de datos, se captó 500 participantes en los tres centros hospitalarios; se incluyó todos los cuestionarios administrados. De los cuestionarios aplicados, 214 (42,8\%) fueron del Hospital Nacional Arzobispo Loayza, 200 (40\%) del Hospital Nacional Edgardo Rebagliati y 86 (17,2\%) de una clínica privada. De los encuestados, 282 $(56,4 \%)$ fueron mujeres, el promedio de edad fue 43,6 $\pm 15,4$ años, con un rango de edad de 18 a 86 años; el $75 \%$ de los participantes tuvo menos de 56 años. No se encontró diferencia significativa en el género, pero sí hubo diferencia significativa en edad y el nivel de educación entre los tres centros hospitalarios (Tabla 1).

El promedio de puntaje alcanzado de nivel de conocimiento fue $5,21 \pm 1,99$; los participantes del Hospital Rebagliati obtuvieron un puntaje promedio de 5,68 \pm 2,05, los del Hospital Loayza, 4,91 \pm 1,79, y los de la clínica privada, $4,87 \pm 2,13$. Se muestra en la Figura 1 los porcentajes de niveles de conocimientos alcanzados por los participantes en forma total y por centro hospitalario. Se encontró diferencia estadísticamente significativa del puntaje promedio y el nivel de conocimientos entre los tres hospitales $(p<0,001)$, usando chi cuadrado y anova respectivamente. El puntaje mínimo alcanzado fue de $0 \mathrm{y}$ máximo de 11 ; el $75 \%$ de pacientes tuvo un puntaje menor de 7 .

El 60,4\% de participantes supo que el internista trata enfermedades no quirúrgicas del adulto y $77,8 \%$ que el internista puede atender tanto en consultorio como en salas de hospitalización. El 59,6\% de participantes supo que para ser internista se requiere al menos 3 años de entrenamiento luego de graduarse como médico, 51,6\% supo que el internista estaría capacitado para cuidar pacientes en unidad de cuidados intensivos (UCI), $57,8 \%$ supo que el internista está entrenado para atender problemas de salud de la mujer adulta y solo $30,6 \%$, que el internista no está entrenado para atender pacientes menores de 17 años. Un dato importante es el $82,4 \%$ de pacientes que consideró que la especialidad de medicina interna es requisito para poder realizar otras especialidades, como cardiología, gastroenterología o neumología. Además, gran parte de los participantes confundió al internista con el médico de familia (57,2\%), con el médico general $(65,4 \%)$ e incluso con el interno de medicina (56,2\%). En la Tabla 2, se detalla las preguntas de conocimientos con el porcentaje de acierto por centro hospitalario. No se encontró asociación del nivel de conocimiento con el género, pero sí del nivel de conocimiento con el nivel educativo. Así, se observó que los pacientes con educación superior tenían mayor

Tabla 1. Datos demográficos de participantes.

\begin{tabular}{lccccc}
\hline & $\begin{array}{c}\text { Hospital } \\
\text { Rebagliati } \\
(\mathrm{n}=200)\end{array}$ & $\begin{array}{c}\text { Hospital } \\
\text { Loayza } \\
(\mathrm{n}=214)\end{array}$ & $\begin{array}{c}\text { Clínica } \\
\text { privada } \\
(\mathrm{n}=86)\end{array}$ & $\begin{array}{c}\text { Total } \\
(\mathrm{n}=500)\end{array}$ & \\
\hline Característica & \multicolumn{5}{c}{ Media \pm DS o Frecuencia $(\%)$} \\
\hline Edad & $47,6 \pm 16,1$ & $41,5 \pm 13,7$ & $39,7 \pm 16,0$ & $43,6 \pm 15,4$ & $<0,001^{*}$ \\
Género masculino & $87(43,5 \%)$ & $86(40,2 \%)$ & $41(47,7 \%)$ & $214(42,6 \%)$ & $0,48^{\dagger}$ \\
Educación superior & $113(56,5 \%)$ & $86(40,2 \%)$ & $73(84,9 \%)$ & $272(54,5 \%)$ & $<0,001^{\dagger}$ \\
\hline
\end{tabular}

* Se aplica Anova.

† Chi cuadrado. 


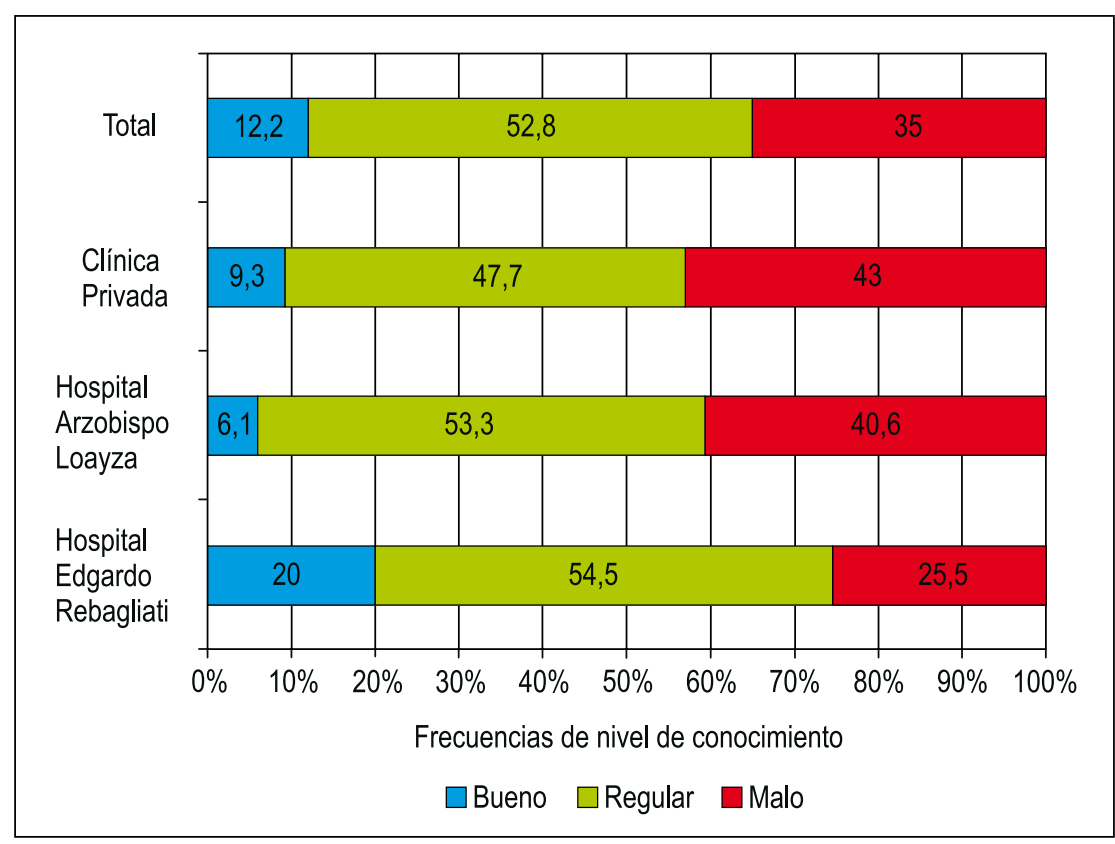

Figura 1. Características del nivel de conocimiento en los tres centros asistenciales.

probabilidad de obtener un buen nivel de conocimiento $(p=0,001, O R=2,72$ $[1,47-5,03])$.

Los participantes del Hospital Rebagliati tuvieron mayor probabilidad de obtener un buen nivel de conocimiento que los participantes del Hospital Loayza
(OR: 3,87 , IC (95\%) $[1,92-7,90])$ y que los pacientes de la clínica privada (OR: 2,44, IC (95\%) [1,03 - 5,94]). No hubo diferencias significativas entre los pacientes del Hospital Loayza y de la clínica privada en cuanto al nivel de conocimiento (OR: 0,63, IC (95\%) [0,23 $-1,74])$.
Se encontró que los pacientes con educación superior tuvieron mayor probabilidad de saber que el médico internista es un profesional distinto al médico general $(\mathrm{OR}=1,50,[1,03-2,20])$, al médico de familia $(O R=1,78,[1,23-2,56])$ y al interno de medicina $(\mathrm{OR}=2,84,[1,95-4,13])$.

Siguiendo con el análisis individual por pregunta o enunciado, los encuestados del Hospital Loayza confundieron con más frecuencia al internista con el médico de familia, que los pacientes del Rebagliati y de la clínica privada $(\mathrm{OR}=1,71$, IC (95\%) [1,15 - 2,54]) y (OR: 2,32, IC (95\%) [1,39-3,87]), respectivamente. Existió también en los participantes del Hospital Loayza mayor confusión con respecto a diferenciar al internista con el interno de medicina, que en los pacientes del Rebagliati y de la clínica privada $(\mathrm{OR}=2,47$, IC $(95 \%)$ $[1,65-3,68])$ y $(\mathrm{OR}=2,24$, IC $(95 \%)$ $[1,34-3,75])$, respectivamente. Ante la pregunta sobre el enfoque integral del internista, los pacientes del Loayza son quienes respondieron menos correctamente, en comparación a los pacientes del Rebagliati (OR=1,71, IC (95\%) [1,15 $-2,54])$ y de la clínica privada $(\mathrm{OR}=3,35$, IC (95\%) $[1,99-5,66])$. Ante la pregunta sobre la capacidad del internista para

Tabla 2. Nivel de conocimientos sobre la medicina interna y el médico internista por centro hospitalario.

\begin{tabular}{|c|c|c|c|c|}
\hline & $\begin{array}{l}\text { Hospital } \\
\text { Rebagliati } \\
(n=200)\end{array}$ & $\begin{array}{l}\text { Hospital } \\
\text { Loayza } \\
(n=214)\end{array}$ & $\begin{array}{c}\text { Clínica } \\
\text { privada } \\
(n=86)\end{array}$ & \\
\hline Enunciado & \multicolumn{3}{|c|}{$\begin{array}{l}\text { Porcentaje de participantes que } \\
\text { respondieron correctamente }\end{array}$} & $\begin{array}{l}\text { Valor } \\
p^{*}\end{array}$ \\
\hline \multicolumn{5}{|l|}{ Preguntas cuya respuesta correcta es VERDADERO } \\
\hline Un médico internista se especializa en enfermedades no quirúrgicas del adulto. & $60 \%$ & $63,1 \%$ & $54,7 \%$ & 0,397 \\
\hline Un médico internista puede atender en consultorio y en salas de hospitalización. & $82 \%$ & $77,6 \%$ & $68,6 \%$ & 0,044 \\
\hline Un médico internista estaría capacitado para cuidar pacientes delicados en Cuidados Intensivos. & $55,5 \%$ & $51,9 \%$ & $41,9 \%$ & 0,106 \\
\hline Un médico internista requiere al menos 3 años de entrenamiento después de graduarse de médico. & $59,5 \%$ & $64,5 \%$ & $47,7 \%$ & 0,027 \\
\hline Un médico internista está usualmente entrenado para atender problemas de salud de la mujer adulta. & $72,5 \%$ & $59,3 \%$ & $19,8 \%$ & $<0,001$ \\
\hline \multicolumn{5}{|l|}{ Preguntas cuya respuesta correcta es FALSO } \\
\hline $\begin{array}{l}\text { Medicina Interna es requisito o requerida para entrar a especialidades como cardiología, } \\
\text { gastroenterología o neumología. }\end{array}$ & $20 \%$ & $14,5 \%$ & $19,8 \%$ & 0,286 \\
\hline Médico internista es otra forma de llamar al médico de familia. & $47 \%$ & $34,1 \%$ & $54,7 \%$ & 0,002 \\
\hline Médico internista es otra forma de llamar al médico general. & $38 \%$ & $33,2 \%$ & $30,2 \%$ & 0,379 \\
\hline Médico internista es otra forma de llamar al interno de medicina. & $53,5 \%$ & $31,8 \%$ & $51,2 \%$ & $<0,001$ \\
\hline Médico internista está capacitado para atender pacientes menores de 17 años. & $33 \%$ & $26,6 \%$ & $34,9 \%$ & 0,238 \\
\hline El médico internista se enfoca en enfermedades específicas más que en la salud integral del paciente. & $47,5 \%$ & $34,6 \%$ & $64 \%$ & $<0,001$ \\
\hline
\end{tabular}

* Prueba chi cuadrado. 
atender problemas de salud de la mujer adulta, fueron los pacientes del Hospital Rebagliati, los que mejor respondieron comparados con los pacientes del Hospital Loayza (OR=1,81, IC (95\%) [1,17 $-2,79])$ y de la clínica privada $(O R=10,7$, IC (95\%) $[5,56-20,80])$.

Los pacientes en general tienen más confianza en el internista para el diagnóstico y manejo de síntomas que para el manejo de enfermedades específicas o para realizar determinadas actividades del quehacer médico. El 79\% de respuestas de los pacientes (2 370 respuestas indicaban confianza para síntomas de 3000 preguntas posibles para los 500 pacientes) indicó confianza en el manejo de síntomas por el internista; 50,26\% (1508 de 3000 ) de las respuestas indicó confianza en la habilidad del internista para realizar actividades clínicas; y 45,9\% (2 985 de 6500 ) de las respuestas mostró confianza en el internista para afrontar y tratar enfermedades específicas. El porcentaje de confianza de los pacientes para cada situación y dividido por centro hospitalario se muestra en el Tabla 3.

No se encontró relación estadísticamente significativa entre la percepción de confianza para el internista al tratar síntomas, enfermedades o para realizar actividades clínicas, con el nivel educativo y género. Sin embargo, sí se encontró relación de la percepción de confianza para el internista en los tres ítems ya mencionados con el hospital de procedencia y el nivel de conocimiento; la percepción de confianza en el internista para el manejo de síntomas fue significativamente mayor en pacientes del H. Rebagliati y en aquellos con buen nivel de conocimiento sobre el internista; la percepción de confianza en el internista para manejo de enfermedades fue significativamente mayor en pacientes del Hospital Loayza y mayor en aquellos con buen nivel de conocimiento sobre el internista. Finalmente, la percepción de confianza en el internista para procedimientos y habilidades clínicas fue significativamente mayor en pacientes del Hospital Rebagliati y en aquellos con regular conocimiento sobre el internista.

Al realizar el análisis multivariado por regresión logística ajustado para
Tabla 3. Percepción de los pacientes sobre la capacidad del internista en problemas médicos y habilidades.

\begin{tabular}{ccccc}
\hline & $\begin{array}{c}\text { Hospital } \\
\text { Rebagliati } \\
(n=200)\end{array}$ & $\begin{array}{c}\text { Hospital } \\
\text { Loayza } \\
(n=214)\end{array}$ & $\begin{array}{c}\text { Clínica } \\
\text { privada } \\
(n=84)\end{array}$ & $\begin{array}{c}\text { Total } \\
(n=500)\end{array}$ \\
\hline Problema médico & \multicolumn{5}{c}{$\begin{array}{c}\text { Porcentaje de pacientes con confianza } \\
\text { en la capacidad del internista }\end{array}$} & $\begin{array}{c}\text { Valor } \\
p^{*}\end{array}$ \\
\hline
\end{tabular}

\section{Síntomas}

Dolor abdominal

Dolor de cabeza

Fatiga

Dificultad para respirar

Dolor torácico

Dolor articular

$\begin{array}{rrrrr}91,5 & 86 & 84,9 & 88 & 0,140 \\ 90 & 84,1 & 74,4 & 84,8 & 0,002^{\dagger} \\ 86 & 84,6 & 62,8 & 81,4 & <0,001^{\dagger} \\ 83,5 & 72,9 & 62,8 & 75,4 & 0,001^{\dagger} \\ 80,5 & 69,2 & 73,3 & 74,4 & 0,022^{\dagger} \\ 75,5 & 68,7 & 60,5 & 70 & 0,037^{\dagger}\end{array}$

\section{Enfermedad}

Hipertensión arterial

Hipercolesterolemia

Lesión por deporte

Infección urinaria

Diabetes mellitus

Neumonía

Depresión

Osteoporosis

Problema de la tiroides

Infección por VIH (sida)

Enfermedad coronaria

Insuficiencia cardiaca

Accidente cerebrovascular

$\begin{array}{rrrrc}77 & 76,2 & 663 & 74,8 & 0,207 \\ 70 & 69,6 & 54,7 & 67,2 & 0,022^{\dagger} \\ 58,5 & 70,6 & 53,5 & 62,8 & 0,006^{\dagger} \\ 55,5 & 65,9 & 38,4 & 57 & <0,001^{\dagger} \\ 52 & 50,5 & 43 & 49,8 & 0,355 \\ 47 & 551 & 36 & 48,6 & 0,010^{\dagger} \\ 45 & 48,1 & 27,9 & 43,4 & 0,005^{\dagger} \\ 39,5 & 39,3 & 27,9 & 37,4 & 0,154 \\ 32 & 36,4 & 27,9 & 33,4 & 0,360 \\ 31 & 38,3 & 23,3 & 32,8 & 0,039^{\dagger} \\ 29,5 & 35,5 & 29,1 & 32 & 0,345 \\ 30 & 34,1 & 24,4 & 30,8 & 0,261 \\ 27.5 & 29 & 19,8 & 26,8 & 0,293\end{array}$

Habilidad o competencia clínica Vacunación

Evaluación pre quirúrgica

Examen manual de próstata

Examen de mama

Inicio de controles prenatales

Examen ginecológico

\begin{tabular}{rrrrl}
71,5 & 81,3 & 80,2 & 77,2 & 0,092 \\
68 & 62,6 & 46,5 & 62 & $0,002^{\dagger}$ \\
51,5 & 52,3 & 33,7 & 48,8 & $0,007^{\dagger}$ \\
44,5 & 46,7 & 34,9 & 43,8 & 0,182 \\
48 & 36 & 36 & 40,8 & $0,019^{\dagger}$ \\
31 & 31,3 & 18,6 & 29 & 0,060 \\
\hline
\end{tabular}

* Prueba chi cuadrado.

${ }^{\dagger}$ Estadísticamente significativo $(p<0,05)$.

género, nivel de educación, hospital de procedencia y nivel de conocimiento, se encontró que los participantes con nivel de conocimiento malo y regular tenían menor probabilidad de tener percepción de confianza en el internista para el manejo de síntomas y enfermedades que los participantes con buen nivel de conocimiento. Además, se observó que los participantes del Hospital Loayza tenían mayor probabilidad de tener percepción de confianza para el manejo de enfermedades y habilidades clínicas que los pacientes del Hospital Rebagliati y de la clínica privada; mientras que los pacientes del Rebagliati tuvieron mayor probabilidad de tener percepción de confianza para el manejo de síntomas que los pacientes del Loayza y de la clínica privada (Tabla 4).

Se encontró algunas asociaciones interesantes en el presente estudio, aplicando chi ${ }^{2}$ y odds ratio. Así, encontramos que los pacientes que no supieron que el internista requiere al menos 3 años de entrenamiento luego de graduado como médico tuvieron mayor desconfianza en el internista para el manejo de síntomas $(p=0,009, O R=1,71[1,14-2,59])$ y en sus habilidades clínicas $(p=0,01$, $\mathrm{OR}=1,63[1,12-2,38]$ ), pero no se encontró asociación con la confianza al internista para el manejo de enfermedades $(p=0,10, O R=1,35[0,03-1,95])$. 
Tabla 4. Análisis multivariado de los factores que influyen en la percepción de confianza hacia el internista*.

\begin{tabular}{lccc}
\hline Variables & $\begin{array}{c}\text { Percepción de } \\
\text { confianza en el } \\
\text { manejo de sintomas } \\
\text { OR }(\text { IC 95\%) }\end{array}$ & $\begin{array}{c}\text { Percepción de } \\
\text { confianza en el manejo } \\
\text { de enfermedades } \\
\text { OR }(\text { IC 95\%) }\end{array}$ & $\begin{array}{c}\text { Percepción de } \\
\text { confianza en } \\
\text { habilidades clínicas } \\
\text { OR (IC 95\%) }\end{array}$ \\
\hline $\begin{array}{l}\text { Hospital donde recibe atención } \\
\text { Loayza (Minsa) }\end{array}$ & $2,09(1,16-3,77)^{\dagger}$ & $2,68(1,48-4,86)^{\dagger}$ & $2,20(1,21-4,02)^{\dagger}$ \\
$\begin{array}{l}\text { Rebagliati (EsSalud) } \\
\text { Clínica privada }\end{array}$ & $2,57(1,40-4,70)^{\dagger}$ & $1,72(0,95-3,11)$ & $2,13(1,18-3,85)^{\dagger}$ \\
$\begin{array}{l}\text { Nivel de conocimiento } \\
\text { Malo }\end{array}$ & 1 & 1 & 1 \\
Regular & $0,23(0,08-0,64)^{\dagger}$ & $0,38(0,20-0,72)^{\dagger}$ & $0,61(0,32-1,16)$ \\
Bueno & $0,31(0,11-0,82)^{\dagger}$ & $0,45(0,25-0,81)^{\dagger}$ & $1,04(0,57-1,87)$ \\
\hline
\end{tabular}

* El modelo de regresión incluyó las variables: género, nivel de conocimiento, hospital de procedencia y nivel educativo.

${ }^{\dagger}$ Estadísticamente significativo para un $p<0,05$, IC 95\%.

Los pacientes que desconocían que el internista está entrenado para afrontar problemas médicos de la mujer adulta tuvieron mayor grado de desconfianza en la habilidad del internista para realizar el examen pélvico ginecológico y examen de mama $(p=0,002, O R=1,87[1,24$ $2,82]),(p<0,001, \mathrm{OR}=2,08[1,44-3,01])$, respectivamente. Sin embargo, no hubo asociación entre género y percepción de confianza al internista para realizar examen pélvico, de mama y examen manual de próstata $(p>0,05)$.

\section{DISCUSIÓN}

En el presente estudio, se encontró que solo $12,2 \%$ de pacientes alcanzó un buen nivel de conocimiento, siendo los pacientes del Hospital Rebagliati perteneciente al Seguro Social los que tenían un mejor nivel de conocimiento, comparado con los pacientes del Hospital Loayza, que pertenece al Ministerio de Salud, y la clínica privada. Sin embargo, al comparar el nivel de conocimiento entre el Hospital Loayza y la clínica privada, no hubo diferencias significativas.

Al comparar nuestros resultados con el estudio de Salerno y col. ${ }^{(1)}$, encontramos mayor confusión en nuestro medio con el concepto de médico internista y otros términos afines, ya que más de la mitad de pacientes confunde al internista con el médico de familia, médico general y con el interno de medicina, mientras que en el estudio de Salerno solo 45\% de pacientes confundía al internista con el médico de familia y médico general, y un porcentaje aún menor lo confundía con el interno de medicina (19\%). Al preguntar sobre el campo de acción del internista, es decir "el tratar enfermedades no quirúrgicas del adulto", la mayoría respondió correctamente $(60,4 \%)$, porcentaje similar al encontrado por Salerno; sin embargo, Lewis y col, en su estudio sobre preferencias de los pacientes por el cuidado del internista, encontró que 96\% de pacientes supo que el internista trata adultos y el $83 \%$ supo que el internista no realiza tratamiento quirúrgico de patologías que lo ameriten ${ }^{(10)}$.

Referente al carácter de especialidad de la medicina interna y que se requiere al menos 3 años de entrenamiento luego de graduarse como médico general, se encontró niveles de conocimiento similares frente a lo encontrado por Salerno (59\%); sin embargo, a pesar que la mayoría de participantes consideró el carácter de especialidad de la medicina interna, gran parte de nuestros participantes $(82,4 \%)$ consideró que la especialidad de medicina interna es requisito para poder realizar otras especialidades, como cardiología, gastroenterología o neumología, cuando sabemos que no es así, ya que la medicina interna es una especialidad distinta a otras y que no es requisito para el realizar otras especialidades distintas. Salerno encontró que 64\% de participantes de su estudio consideró como verdadero este enunciado. Por otro lado, Lewis halló que casi la mitad de pacientes consideró que de 3 a 4 años era la duración del entrenamiento para médico general y la mayoría, que tomaba de 7 a 9 años ser especialista.

En el estudio de Salerno, la mitad de participantes supo que el internista está entrenado para atender problemas de salud de la mujer adulta, mientras en nuestro estudio se encontró un mayor porcentaje de acierto $(57,8 \%)$. Sobre la capacidad del internista para atender pacientes menores de 17 años, Salerno encontró 36\% de pacientes que pensó que el internista no está entrenado para este grupo etáreo, porcentaje similar encontrado por nuestro estudio; lo que difiere de lo encontrado por Lewis, quien informó en su estudio que 51\% de sus participantes consideró al internista como entrenado para atender niños. En una revisión sobre medicina del adolescente, se menciona que los internistas están relacionados y capacitados con muchos de los problemas que afectan a los adolescentes, como dolor abdominal, lesiones deportivas y temas reproductivos; sin embargo, algunos problemas de los adolescentes son menos familiares para los internistas, como los problemas del comportamiento (déficit de atención/ desórdenes de hiperactividad, desórdenes de conducta), problemas nutricionales (anorexia nervosa y bulimia nervosa) y desórdenes del crecimiento y desarrollo puberal ${ }^{(11)}$.

En nuestro estudio, encontramos que los pacientes con nivel educativo superior tenían mejor nivel de conocimiento; no encontramos tal relación en el estudio de Salerno y Lewis. Sin embargo, Salerno halló que los pacientes con educación superior tenían más probabilidad de conocer la diferencia entre internista con interno de medicina y médico de familia. Al realizar la misma asociación, encontramos que, efectivamente, los pacientes con educación superior tenían más probabilidad de saber que el internista es un profesional distinto al interno de medicina, médico de familia y médico general.

En cuanto a la percepción de los pacientes sobre el rol y desempeño del 
médico internista, encontramos que los participantes confiaban más en el internista para el manejo de síntomas, seguido de la percepción de confianza en las habilidades clínicas del internista y por último un menor nivel de confianza para el manejo de enfermedades específicas. Salerno encontró una percepción similar, con un $76 \%$ de respuestas que indicaban confianza en las habilidades del internista para el manejo de síntomas, 59\% de respuestas con confianza en el manejo de enfermedades y $54 \%$ de respuestas con confianza en las habilidades clínicas del internista.

Un hallazgo importante fue la asociación entre el nivel de conocimiento alcanzado y la percepción de confianza, puesto que un nivel de conocimiento regular o malo estuvo relacionado con menor probabilidad de percepción de confianza en el internista para el manejo de síntomas y enfermedades; es decir, el tener buen nivel de conocimiento sobre lo que es la medicina interna y el ámbito de acción del internista produciría mayor confianza en la labor y desempeño del médico internista. Otra asociación importante fue la hallada entre el hospital de procedencia y la percepción de confianza; los pacientes del Loayza tuvieron mayor probabilidad de tener percepción de confianza para el manejo de enfermedades y el llevar a cabo actividades o exámenes clínicos por parte de internista, mientras que en los pacientes del Rebagliati se encontró también una mayor probabilidad de tener percepción de confianza para el internista en el manejo de síntomas.

En nuestro estudio, no hallamos relación entre género y nivel educativo con la percepción de confianza para el internista, lo cual difiere de lo hallado por Salerno, quien encontró que los pacientes con educación superior tuvieron significativamente más confianza en el internista para el manejo de enfermedades y síntomas.

Lewis halló que solo una minoría de pacientes prefiere acudir al especialista según el órgano o sistema afectado para su tratamiento; por ejemplo, solo 3\% de los pacientes consideró acudir a un especialista distinto del médico internista para el manejo de hipertensión arterial o hipercolesterolemia, $13 \%$ para el manejo de diabetes mellitus y $14 \%$ para el manejo de infección del tracto urinario.

Algo similar ocurre en nuestro medio y en el estudio de Salerno, ya que las 4 enfermedades que gozan con una mejor percepción de confianza en el manejo del internista fueron hipertensión arterial, infección urinaria, hipercolesterolemia y diabetes mellitus. En ese sentido, el estudio de Lewis concluye que, para los pacientes, el internista es capaz de tratar la mayoría de condiciones o síntomas comunes y que los pacientes prefieren acudir al internista para recibir tratamiento de condiciones médicas crónicas o conocidas y para la mayoría de las condiciones nuevas ${ }^{(10,12)}$.

En nuestro estudio, los pacientes tuvieron una menor percepción de confianza para el diagnóstico y manejo de enfermedades, siendo las enfermedades con menos percepción de confianza accidente cerebrovascular, insuficiencia cardiaca, enfermedad coronaria, infección por VIH-sida y problemas de la tiroides. Dentro de las habilidades médicas con menor percepción de confianza estuvieron el examen pélvico ginecológico, seguido por el Inicio de controles prenatales. Salerno encontró que los internistas gozan de menos percepción de confianza para el manejo de lesiones de deporte (41\%), infección por VIH-sida (46\%), osteoporosis $(46 \%)$, insuficiencia cardiaca ( $47 \%)$ y depresión (48\%).

Lewis, por su parte, halló que para el inicio de controles prenatales, problemas de próstata y problemas odontológicos, un mayor porcentaje de pacientes prefirió acudir con un especialista distinto al internista. Respecto a esto, una revisión sobre la interfase entre el manejo del internista y otras especialidades, nos dice que el flujo de pacientes entre internistas y otras especialidades se ha incrementado y que el médico internista manejaría mejor pacientes con enfermedades crónicas y complejas ya definidas y diagnosticadas, mientras que las demás especialidades más definidas en su ámbito (cardiología, reumatología, neumología) deberían enfocarse a procedimientos de su experticia, manejo inicial o agudo de los pacientes (12). Sin embargo, estas recomendaciones son desconocidas por la mayoría de pacientes.

Al relacionar género con la percepción de confianza para el examen de mama, pélvico y examen manual de próstata, no encontramos alguna asociación; sin embargo, Salerno halló que las mujeres tenían confianza por el internista para realizar examen de mama y pélvico y que los varones tuvieron confianza en el internista para el examen manual de próstata.

Como sabemos, la percepción de los pacientes ha ganado creciente importancia en la práctica y el negocio de la medicina, y hay un creciente sentimiento que si los internistas establecen una reputación pública de experticia en medicina de adultos, sería más efectivo en la atracción y retención de pacientes ${ }^{(2)}$. En 1996, la American College of Physicians-American Society of Internal Medicine (ACP-ASIM), percibió la necesidad de educar a los pacientes sobre la especial experticia en adultos de los médicos internistas ${ }^{(1)}$. Para esto, realizaron una encuesta telefónica en 1000 personas seleccionadas al azar, evaluando la percepción del público sobre la labor de los internistas. La encuesta encontró que solo para $18 \%$ de participantes los internistas podrían actuar como médicos de primer nivel de atención, mientras $56 \%$ sabía que los internistas eran especialistas, porcentaje similar a lo encontrado en nuestro estudio. Además, solo casi un tercio de los pacientes se sintió confortable con un internista tratando problemas específicos de la mujer, como osteoporosis, tamizaje de cáncer cervical y examen de las mamas ${ }^{(1,5)}$; datos que también son parecidos a los encontrados en nuestro estudio y en el de Salerno ${ }^{(1)}$. Estos resultados ayudaron al ACP-ASIM a organizar una campaña publicitaria, con el titular "campaña de relaciones públicas por la medicina interna", en la que se informaría sobre qué son y qué hacen los internistas y por qué ellos son los más calificados en atender adultos. La ACP-ASIM retuvo el nombre tradicional de la especialidad (medicina interna) en el anuncio de la campaña, pero decidió unirle la siguiente frase descriptica 'Doctores para adultos' ${ }^{(1)}$. La campaña puso énfasis en que el internista es el especialista con un estudio de posgrado de tres 
años en la prevención y tratamiento de enfermedades que afectan a los adultos, entre los 36 y 60 años ${ }^{(1,6)}$. Campañas de educación de este tipo no han sido realizadas en nuestro medio y, como vemos luego del análisis de los resultados del presente estudio, es evidente una puesta en marcha de una campaña similar. Si en los Estados Unidos, donde la medicina interna es la mayor especialidad con cerca de 200000 internistas de 800000 médicos y en donde se ven numerosas presiones ejercidas que la fuerzan a un cambio continuo, existe desinformación y no se tiene una idea clara, completa y segura de lo que es la medicina interna y el internista, es lógico suponer que en nuestro país el nivel de desinformación es aún mayor. Sin embargo, en nuestro estudio encontramos que los niveles de conocimiento y de percepción de confianza son similares a los encontrados en otros estudios realizados en los Estados Unidos.

No solo es el desconocimiento del público; existen otros factores que van en contra de la medicina interna, como la disminución de interés de los estudiantes y residentes en la medicina interna (entre 1985 y 1993, el número de graduados, en los Estados Unidos, luego de 3 años de residencia para medicina interna, declinó en más de 30\% (13)), la falta de satisfacción entre los internistas que dejan la medicina interna a una tasa alarmante, el aumento del costo del cuidado de la salud y el rápido envejecimiento de la población. Estas situaciones adversas deberían hacer que la medicina interna fuera proactiva en influir sobre el sistema de cuidado de salud en el futuro, así como fortalecerse como especialidad ${ }^{(9,14)}$. Para ello, los resultados del presente estudio ayudarían a los médicos internistas a conocer la percepción de los pacientes sobre las patologías de la que gozan mayor confianza y desarrollar una educación médica continua sobre tales cuadros, además de dar a conocer a los internistas acerca de los déficits en el conocimiento de los pacientes sobre la medicina interna y ayudar en forma progresiva a ellos en conocer mejor y despejar dudas sobre qué es la medicina interna y el ámbito de acción del internista.

En resumen, solo $12 \%$ de pacientes obtuvo un buen nivel de conocimiento respecto a lo que es la medicina interna $\mathrm{e}$ internista, siendo los pacientes del Hospital del Seguro Social los que tuvieron mejor nivel de conocimiento; con el análisis multivariado y obteniendo $\mathrm{OR}$ ajustados encontramos que solo el nivel de conocimiento y el hospital de procedencia se relacionaron con la percepción de confianza de los pacientes. Otra conclusión importante es que los pacientes tuvieron mejor percepción de confianza para el manejo de síntomas por parte del internista, seguido de la confianza en las habilidades clínicas y por último para el manejo de enfermedades.

Se recomienda, por tanto, tomar las medidas correctivas al respecto, ya que el desconocimiento sobre lo que exactamente es la medicina interna y sobre el rol del internista no solo va en detrimento de la especialidad y del especialista, sino también del propio paciente, ya que desconoce en el internista a un profesional médico especialista que tiene un enfoque integral del paciente adulto ${ }^{(6)}$. El reto de la medicina interna es demostrar y 'empoderar' a los adultos que la atención por el internista conlleva a resultados para la salud de la más alta calidad y también a una adecuada relación costo beneficio y tiempo beneficio.

\section{AGRADECIMIENTOS}

A la Licenciada Paola Pérez Rojas por la ayuda logística y coordinaciones en la aplicación del cuestionario. Al Dr. Cesar Gutiérrez, Médico Epidemiólogo, Profesor de la Sección Epidemiología y Estadística, Facultad de Medicina de San Fernando, UNMSM, por la ayuda en la presentación de los resultados del presente trabajo.

\section{REFERENCIAS BIBLIOGRÁFICAS}

1. Salerno SM, Landry FJ, Kaboli PJ. Patient perceptions of the capabilities of internists: a multi-center survey. Am J Med. 2001;110(2):1117.
2. Goldman L. Adult (not internal) medicine. Ann Intern Med. 1997;127(9):835-6.

3. Reyes H. What is Internal Medicine? Rev Med Chile. 2006;134:1338-44.

4. Espinoza A. Medicina Interna ¿Qué fuiste, Qué eres, Qué serás? Rev Cubana Med. 1999;38(1):7990.

5. Arneson J, McDonald WJ. Can we educate the public about internal medicine? Initial results. Am J Med. 1998;105:1-5.

6. Pamo 0. Medicina Interna: Medicina para los adultos. Bol Soc Per Med Interna. 1999;12(2):124.

7. Generating more generalists: an agenda of renewal for internal medicine. Federated Council for Internal Medicine. Ann Intern Med. 1993;119(11):11259.

8. Varela. N. Ejercicio de la Medicina Interna en América Latina. Rol del médico internista. Rev Med Chile. 2002;130(1):96-100.

9. Sox. H, Scott. D, Ginsburg. J. The role of the future general internist defined. American College of Physicians. Ann Intern Med. 1994;121(8):61622.

10. Lewis CL, Wickstrom GC, Kolar MM, Keyserling TC, Bognar BA, Dupre CT. Patients preferences for care by general internists and specialists in the ambulatory setting. J Gen Intern Med. 2000;15:7583.

11. Schmidt RM, White LK. Internists and adolescent medicine. Arch Intern Med. 2002;162:1550-6.

12. Linzer M, Myerburg R, Kutner J, Wilcox M, Oddone $\mathrm{E}$, DeHoratius RJ, et al. Exploring the generalistsubspecialist interface in internal medicine. Am J Med. 2006;119(6):528-37.

13. McMurray JE, Schwartz. MD, Genero NP, Linzer $M$. The attractiveness of internal medicine: a qualitative analysis of the experiences of female and male medical students. Society of General Internal Medicine Task Force on Career Choice in Internal Medicine. Ann Intern Med. 1993;119(8):812-8.

14. Hemmer PA, Costa ST, DeMarco DM, Linas SL, Glazier DC, Schuster BL. Predicting, preparing for, and creating the future: what will happen to internal medicine? Am J Med. 2007;120(12):1901-6.

Manuscrito recibido el 13 de febrero de 2009 y aceptado para publicación el 10 de marzo de 2009.
Correspondencia:
Dr. Franco Romaní Romaní
Av. Héroes del Pacifíco 615. Chorrillos
Lima 9, Perú
Correo-e: frrr812@hotmail.com 